\title{
An Event-Based Empirical Process Analysis Framework
}

\author{
Wikan Danar Sunindyo, Stefan Biffl, Richard Mordinyi, Thomas Moser, Alexander Schatten, \\ Mohammed Tabatabai, Dindin Wahyudin, Edgar Weippl, Dietmar Winkler \\ Christian Doppler Laboratory for Software Engineering Integration for Flexible Automation Systems \\ Vienna University of Technology, Favoritenstrasse 9-11/188 1040 Vienna, Austria
}

\{wikan, biffl, mordinyi, moser, schatten, tabatabai, dindin, weippl, winkler\}@ifs.tuwien.ac.at

\begin{abstract}
The engineering of complex software-intensive systems, like industrial production plants, requires software engineering to coordinate and interact with other engineering disciplines. Project and quality managers need empirical study results to improve system quality, e.g., from process analysis of engineering process event sequences. In this paper, we propose a framework adapted from business process analysis to empirically analyzing engineering process event information. Initial results support the suitability of the approach for (software + ) engineering environments.
\end{abstract}

\section{Categories and Subject Descriptors}

D.2.8 [Software Engineering]: Metrics - process metrics.

\section{General Terms}

Management, Measurement, Performance, Design, Verification.

\section{CONTEXT AND MOTIVATION}

The engineering of complex software-intensive systems typically involves the cooperation of different engineering fields, like mechanical, electrical and software engineering [1]. We call these projects "(software + ) engineering" projects as software engineering provides a large proportion of additional value to softwareintensive products and project success depends on the seamless collaboration with non-software engineering fields. A common goal and challenge of project and quality managers is to monitor, control and improve engineering projects across these engineering tools and data models. Empirical study is needed to improve the system quality by collecting and analyzing data from process event data coming from a heterogeneous tool set.

Research approaches on using of events that coming from information system activities as a foundation for process modeling and analysis have been reported for example by van der Aalst et al. [2]. We assume that their approach, called "process mining", which includes process discovery, performance analysis, and conformance checking can be adapted to analyze processes from software projects domain. However, further research is needed to identify and transform the relevant engineering-project-related events.

\section{RESEARCH APPROACH}

Based on the need for monitoring and improving crossdisciplinary engineering projects, we derive two research questions: 1. What adaptations are necessary to use the "process mining" methodology for analyzing (software+) engineering proc- esses? 2. What kinds of events need to be integrated?

We propose a framework for empirical event-based process analysis, as illustrated in Figure 1. The framework consists of three steps: (1) Heterogeneous event data is collected from a variety of tools used by multiple engineering disciplines. (2) Semantic integration integrates the collected heterogeneous event sequences and stores the data in an event log. (3) The integrated event data is used for process mining.

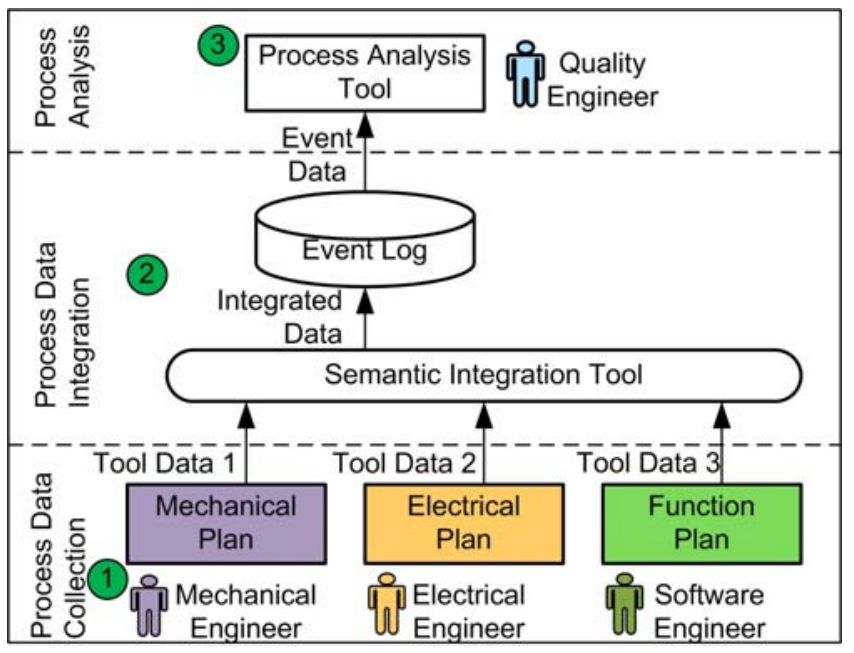

Fig. 1: Framework for event-based engineering process analysis.

We conducted a first pilot application with semantically integrated data models from heterogeneous engineering disciplines at an industry partner. The results show that the proposed framework allows to collect event log data from heterogeneous data sources and analyze the process paths taken in the project. These analyses support process structure discovery and performance analysis. Engineering processes are well compatible to business processes; however, engineering environments often must be adapted to provide all necessary start/stop events to monitor processes correctly.

\section{REFERENCES}

[1] S. Biffl and A. Schatten, "A Platform for Service-Oriented Integration of Software Engineering Environments," in 8th Conference on New Trends in Software Methodologies, Tools and Techniques (SoMeT 09), 2009, pp. 75-92.

[2] W. M. P. van der Aalst, A. J. M. M. Weijters, and L. Maruster., "Workflow Mining: Discovering Process Models from Event Logs," IEEE Transactions on Knowledge and Data Engineering, vol. 16, no. 9, pp. 1128-1142, 2004. 